\title{
Innovative Uses for Conventional Radiation Detectors via Pulse Shape Analysis
}

\author{
J. Kammeraad \\ D. Beckedahl \\ J. Blair \\ A. Friensehner \\ G. Schmid
}

March 3, 1999

This is an informal report intended primarily for internal or limited external distribution. The opinions and conclusions stated are those of the author and may or may not be those of the Laboratory.

Work performed under the auspices of the U.S. Department of Energy by the Lawrence Livermore National Laboratory under Contract W-7405-ENG-48. 


\section{DISCLAIMER}

This document was prepared as an account of work sponsored by an agency of the United States Government. Neither the United States Government nor the University of California nor any of their employees, makes any warranty, express or implied, or assumes any legal liability or responsibility for the accuracy, completeness, or usefulness of any information, apparatus, product, or process disclosed, or represents that its use would not infringe privately owned rights. Reference herein to any specific commercial product, process, or service by trade name, trademark, manufacturer, or otherwise, does not necessarily constitute or imply its endorsement, recommendation, or favoring by the United States Government or the University of California. The views and opinions of authors expressed herein do not necessarily state or reflect those of the United States Government or the University of California, and shall not be used for advertising or product endorsement purposes.

This report has been reproduced directly from the best available copy.

Available to DOE and DOE contractors from the Office of Scientific and Technical Information P.O. Box 62, Oak Ridge, TN 37831

Prices available from (423) 576-8401

Available to the public from the National Technical Information Service

U.S. Department of Commerce 5285 Port Royal Rd., Springfield, VA 22161 
Innovative Uses for Conventional Radiation Detectors via Pulse Shape Analysis Final Report 97-ERD-017

March 3, 1999

J. Kammeraad, D. Beckedahl, J. Blair, A. Friensehner, and G. Schmid

\section{Introduction}

It is well known $[1,2]$ that the leading edge of $\gamma$-ray signals from a semiconductor detector depends upon the particulars of the $\gamma$-ray interaction and the detector itself. The incident $\gamma$-ray interacts at one or more locations in the detector, creating electron-hole pairs that drift under the influence of the imposed electric field to their respective electrodes. The resulting current depends upon the number of charge carriers, the local electric field, and the charge carrier drift velocity.

In most applications, only a measure of the total energy deposited by the $\gamma$-ray is needed. Optimum energy resolution is obtained by shaping the signal, which strongly filters out the variations in the leading edge of the signals. In this project we have shown that by properly recording the signals from a cylindrical high purity germanium (HPGe) detector and utilizing pulse shape analysis, one can obtain the distribution of the deposited energy as a function of the radial coordinate in the detector. In this paper we present predicted and measured position-dependent detector responses, a maximum likelihood approach for accurately determining the radial energy deposition, and a least squares approach for determining the radial energy deposition approximately and 
rapidly enough for real-time applications in Compton suppression. The position resolution that can be obtained in this process depends upon the spectral properties of the electronic noise. We discuss the uncertainties that result from the noise, and show that excellent position resolution can be expected. We also discuss how this kind of signal processing can be extended with segmented HPGe detectors to obtain all three coordinates of $\gamma$-ray interactions, allowing the development of a high efficiency Compton "camera" for $\gamma$-ray imaging.

Analog and digital pulse shape analysis with Ge detectors has been utilized in many instances to optimize detector performance by deriving additional information from pulses beyond a measure of the peak height or integral. Applications include discrimination between $\gamma$-ray, neutrons, and protons [3], identification of beta decays in the detector [4-6], rejection of Compton escape events [7-11], reduction of $\gamma$-ray Doppler broadening effects [12], and adaptive shaping to enhance energy dispersive spectroscopy [13]. Signal processing techniques are also important in the development of detectors for basic nuclear physics research, such as the proposed Gamma-Ray Energy Tracking Array (GRETA) [14].

\section{Determination of the radial position of gamma-ray interactions in a cylindrical HPGe detector}

For the energy range of interest $(\mathrm{E}<2 \mathrm{MeV})$, the ranges of electrons generated by $\gamma$-ray interactions in germanium are small (less than $2 \mathrm{~mm}$ ). Thus to a good approximation, one can consider the signal, $S(t)$, from a multipleinteraction $\gamma$-ray event to be the sum of the signals, $S_{i}(t)$, resulting from the 
energy deposited at the location of each $\gamma$-ray Compton/photoelectric interaction:

$$
S(t)=\sum_{i=1}^{m} S_{i}(t),
$$

where $\mathrm{m}$ is the number of Compton/photoelectric interactions in the event. In a coaxial detector each $S_{i}(t)$, can be expressed as $\operatorname{Si}(t),=E_{i} K\left(t, r_{i}\right)$, where $E_{i}$ is the energy deposited at interaction $i$, and $K\left(t, r_{i}\right)$ is the response of the detector to an impulse of unit energy at radius $r_{i}$. Then Equation (1) becomes:

$$
S(t)=\sum_{i=1}^{m} E_{i} K\left(t, r_{i}\right)=E \sum_{i=1}^{m} a_{i} K\left(t, r_{i}\right)
$$

where $E$ is the total energy deposited in the $\gamma$-ray event and $a_{i}$ is the fractional energy deposited at interaction $i$. For conventional HPGe detectors, the goal is to determine the coefficients $a_{i}$ for each measured signal, thus providing the energy deposition as a function of $r$. The ability to do this in a manner that is useful to various detection applications depends upon a good understanding of the functions $K(t, r i)$, adequate radial resolution (which depends upon both signal and noise properties), and a rapid numerical algorithm to invert Equation (2).

\subsection{Predicted and measured signals as a function of radius.}

Because charge carrier trapping is negligible in high purity germanium, the leading edge signal shape depends only upon the position at which the charge carriers are generated, the local electric field along their drift paths, and their drift velocity. Figure 1 shows several theoretical signals from single interactions in a coaxial detector as a function of radial position. (In these 
calculations we have assumed that the charge carrier velocity is saturated and constant throughout the detector.)

The signals, which represent the induced charge due to electron/hole current, have distinctly different shapes. Each signal changes smoothly as the electrons and holes move into regions of higher and lower electric field respectively. Discontinuities in the derivative of each signal occur at points in time which correspond to the arrival of a group of charge carriers at an electrode.

We measured the impulse response functions $K\left(t, r_{i}\right)$ with a closed-end, coaxial, n-type, high purity germanium (HPGe) detector with an inner radius of $0.5 \mathrm{~cm}$, an outer radius of $2.5 \mathrm{~cm}$, and length of $5 \mathrm{~cm}$. The closed end is $9 \mathrm{~mm}$ thick. The bias voltage applied to the detector is -3000 volts, which is sufficiently high that the drift velocities of the charge carriers can be taken to be saturated and constant over most of the volume of our detector, as described below.

Signals from the detector preamplifier were processed in two parallel paths. In one path the conventional signal shaping and pulse height measurement was performed. In the other path the signal was compensated, amplified, and recorded in digital form. The compensating circuit, consisting of wide-bandwidth amplifiers and RC filters, was selected to extend the system bandwidth to $25 \mathrm{MHz}$ and to condition the noise to make it into "white noise". As described in Section 2.2, this step is necessary in order to use a least-squares approach when solving for the coefficients $a_{i}$ in Eq 2. Two different types of compensating circuits were used in this project. In the initial circuit we used RC filters that differentiated the signals in order to make the discontinuities in slope more apparent. Although this approach is useful for purposes of visualization, it is not the optimum approach. Later, we used filters that compensated for the 
high frequency loss of the preamplifier, which allowed a more accurate determination of the coefficients $a_{i}$ for each measured signal. The signals were recorded every nanosecond with 8 bit accuracy with a Tektronix DSA 602 digitizer, and recorded in event mode along with the usual measurement of the pulse height of the shaped signal. The event mode data were later analyzed on a Macintosh 8100 computer using LabVIEW ${ }^{\circledR}$.

We obtained the radial response functions $K(t, r)$ by two methods: direct measurement using a highly collimated $\gamma$-ray source; and convolution of the idealized signals (as in Fig. 1) with the preamplifier frequency response. The measurement of the preamplifier frequency response is described in Section 2.2.

Measurements of detector response functions $K_{0}(t, r)$ were obtained using a 2-inch long, 1-mm diameter Au collimator in a 6-inch long heavymet holder that attenuated transmitted $\gamma$-rays. The collimator was carefully aligned with the detector and positioned such that the umbra was centered on the radial zone of interest. A Cs-137 check source was placed on the collimator axis, and the signals were gated to record only those events in which the deposited energy was between $0.42 \mathrm{MeV}$ and $0.47 \mathrm{MeV}$; i.e., the event was near the "Compton edge". This energy was chosen because the probability of a single interaction is relatively high in this region. Measurements were obtained at 20 equally spaced locations along a detector radius, each $1 \mathrm{~mm}$ apart.

Even with gating and tight collimation, most measured signals did not represent single interactions at the target radii. Signals were accepted or rejected according to the following method. Each signal $S_{j}(t, r)$ measured at radius $r$ was compared with the signal $K_{p}\left(t, r, v_{e}, v_{h}\right)$ predicted with specific values of the 
electron and hole velocities $v_{e}$ and $v_{h}$. We kept those signals that satisfied the following equation:

$$
\int_{0}^{\mathrm{T}}\left|\mathrm{S}_{\mathrm{j}}\left(\mathrm{t}-\tau_{\mathrm{j}}, \mathrm{r}\right)-\mathrm{c}_{\mathrm{j}} \mathrm{K}_{\mathrm{p}}\left(\mathrm{t}, \mathrm{r}, \mathrm{v}_{\mathrm{e}}, \mathrm{v}_{\mathrm{h}}\right)\right|^{2} \mathrm{dt} \leq \alpha,
$$

where $\mathrm{T}=1024 \mathrm{~ns}$, and the time shift $\tau$ and the normalization constant $c_{j}$ were chosen to minimize the integral. Using $\alpha=5 \times 10^{-5}$, we averaged the signals that satisfied Equation (3) in order to obtain the measured pulse response function at each radial location. The number of signals in the average varied as a function of position from about 50 in the inner zones to about 250 in the outer zones.

The radial responses obtained by the two methods are very similar to each other, as shown in Figure 2 for one response function. The responses obtained by convolution of the ideal signals with the system response were slightly modified, by adjusting the velocities of the charge carriers, to fit the width of the responses obtained by the collimation method. By using an electron drift velocity of $9.5 \times 10^{6} \mathrm{~cm} / \mathrm{s}$ and hole drift velocity of $7.5 \times 10^{6} \mathrm{~cm} / \mathrm{s}$, we were able to obtain good agreement between the responses obtained by both methods for all ten radial zones with our detector.

The high degree of similarity between the two curves in Figure 2 is very encouraging. It implies that for the coaxial portion of the detector the signals can be understood in terms of very simple quantities: the electric field, the energy deposited (i.e., the number of charge carriers generated), and the charge carrier velocities. As a result, the techniques described in this report can probably be extended to other HPGe detectors in a straightforward manner. 
The closed end (non-coaxial or "quasi-planar" region) of the detector introduces a difficulty, because $\gamma$-rays that interact in this region generate signals that are not well fit by Equation (2). In principle one could measure an additional set of response functions which depend upon two cylindrical coordinates, $r$ and $z$, taking into account the possibility that drift velocities may vary with position in this region. This approach adds significant complexity to the inversion process. Alternatively, one could treat the signals that arise from an interaction in the closed end by another strategy, such as rejecting them or counting them in a separate spectrum.

\subsection{Maximum likelihood approach}

The goal is to accurately determine, from observed pulse shapes, the radial positions and energies of the individual $\gamma$-ray interactions. If the pulse shapes were recorded with perfect accuracy, one could calculate the parameter values ( $E_{i}$ and $r_{\mathrm{i}}$ ) in equation (2) that give equality. Fortunately, there is only one solution to this problem. However, the signals are not recorded with perfect accuracy. They are corrupted by electronic noise from the measurement system. The noise is significant, and its magnitude has already been reduced to nearly the lowest practical level. This being the case, it is desirable to estimate the unknown parameter values in a manner that makes good use of the statistics of the noise.

We have chosen to use maximum-likelihood (ML) estimates for the unknown parameters. Chapter 7 of [15] and section 2.4.2 of [16] explain why this 
is a good approach to use. To describe the ML approach we need to rewrite (2) as

$$
y_{j}=S\left(t_{j}\right)=\sum_{i=1}^{m} E_{i} K\left(t_{j}-t_{0}, r_{i}\right)+e_{j},
$$

where we have taken account of the facts that (a) the data is only available at discrete times, $t_{j}$, which are assumed to be equally spaced, (b) there are measurement errors, $e_{j}$, due to the noise in the system, and (c) the time, $t_{0}$, of the interaction is unknown. We can rewrite (4) as

$$
y=a(p)+e,
$$

where $y$ is the vector of signal values, $\boldsymbol{e}$ is the vector of error values, $\boldsymbol{p}$ is the vector of $2 m+1$ parameter values ( $m$ values of $r, m$ values of $E$, and $t_{0}$ ), and $\boldsymbol{a}$ is the vector of the modeled signal in (4), which depends on the parameter values.

To apply the maximum-likelihood method we need the probability density, $P_{N}(e)$, of the noise. This is obtained by calculation or by measurement. Using (5), we rewrite $\boldsymbol{e}$ as $\boldsymbol{y}-\boldsymbol{a}(\boldsymbol{p})$. The maximum-likelihood estimate is then expressed as

$$
\text { Find } \boldsymbol{p} \text { to maximize } P_{N}(\boldsymbol{y}-\boldsymbol{a}(\boldsymbol{p})) \text {. }
$$

When a probability density is written as a function of the data and the unknown parameters, it is called a likelihood function; hence the name, maximum likelihood. A particularly interesting situation occurs when the errors have a Gaussian distribution, all have the same variance, $\sigma^{2}$, and are statistically independent. This gives 


$$
\begin{array}{r}
P_{N}\left(e_{1}, e_{2}, \ldots e_{n}\right)=\prod_{j=1}^{n} \frac{1}{\sqrt{2 \pi \sigma^{2}}} \exp \left[-\frac{e_{j}^{2}}{2 \sigma^{2}}\right] . \\
=\left(2 \pi \sigma^{2}\right)^{-n / 2} \exp \left[-\frac{1}{2 \sigma^{2}} \sum_{j=1}^{n} e_{j}^{2}\right]
\end{array}
$$

Rewriting $e_{j}$ in (7) as a function of the data and the unknown parameters and realizing that maximizing (7) is equivalent to minimizing the summation in the exponent, we obtain

$$
\text { Find } p \text { to minimize } \sum_{j=1}^{n}\left(y_{j}-a_{j}(p)\right)^{2} \text {. }
$$

This is a simple non-linear-least-squares problem, which can be numerically solved [17, pp. 681-688].

For this special case the maximum-likelihood estimate reduces to the least-squares estimate. Our approach is to preprocess the data in such a way that the least-squares estimate always yields a maximum-likelihood estimate. The conditions required for this are that the noise samples be Gaussian, have equal variance, and be statistically independent. For a well-constructed system, the errors are due to thermal noise being passed through a linear, time-invariant system. This fact guarantees [18, p. 189] that the error samples are Gaussian and have equal variance. However, the errors will not normally be statistically independent.

The condition for the errors to be statistically independent is that the noise must be white [18, p. 145-155]. This means that if we can force the noise to be white, without increasing its magnitude relative to the signal, the maximumlikelihood problem will reduce to the least-squares problem. We accomplish this by measuring the power spectrum of the noise and designing a filter to convert 
this spectrum to white. We then apply this filter to the signals that are recorded. For this to work properly, the model signals in (2) have to be the signals that have already been passed through the filter. The filter can be constructed as an analog filter that is applied to the signals before they are digitized, a digital filter, which is applied after the signals are digitized, or a combination of the two.

We end this section with data demonstrating how this was applied to the detector used in the experiments for this project. The frequency response of our preamplifier was measured two different ways, yielding consistent results. It was measured directly with a network analyzer, and it was measured by supplying a step function signal to the test input of the detector. The frequency response was then calculated from the measured step response. We measured the noise output with a spectrum analyzer. We found that the noise power spectrum was nearly equal to the square of the frequency response. This is what would occur if the noise were due to white noise at the input of the preamplifier. The measured frequency response (from the network analyzer) is shown in figure 3.

The $-3 \mathrm{~dB}$ bandwidth of the preamplifier is $8 \mathrm{MHz}$; a bandwidth of 25 $\mathrm{MHz}$ is required to get optimum spatial resolution from the detector. We designed a compensating circuit (i.e. with frequency response the inverse of this) using wide-bandwidth amplifiers and RC circuits. After the compensating circuit was installed, the noise spectrum was again measured. The integral of the noise spectrum is shown in figure 4 .

White noise has a constant power spectrum, so the integral of the spectrum is a straight line. This shows that the noise is very close to being white out to $40 \mathrm{MHz}$, indicating that the least-squares estimates will be maximum- 
likelihood estimates. The noise power has been converted from volts squared to $\mathrm{MeV}$ squared to correspond to the units we are using for the signals. The noise density (the slope of the straight line) is $3.6 \times 10^{-7} \mathrm{MeV}^{2} / \mathrm{MHz}$.

\subsection{Theoretical limits in radial resolution due to noise}

Using the measured noise intensity and the impulse response, one can calculate the uncertainty, due to noise of the least-squares estimates of the unknown parameters. We assume we have measurements satisfying equation (4) and that the errors $e_{j}$ are independent and have standard deviation, $\sigma$, given by

$$
\sigma^{2}=\frac{I}{2 \Delta t}
$$

where $I$ is the noise intensity in $\mathrm{Mev}^{2} / \mathrm{MHz}$, and $\Delta t$ is the time between samples in $\mu \mathrm{S}$. We have found that the data in the frequency range between $1 \mathrm{MHz}$ and $25 \mathrm{MHz}$ control the results, so if the noise is not perfectly white, its intensity in this 1 to $25 \mathrm{MHz}$ range should be used. The measured value for the system on which we did our experiments was $I=3.6 \times 10^{-7}$. The derivation of equation (9) is given in [19].

The parameter values determined by the least-squares solution (6) will contain a random error due to the influence of the noise. This error will have a mean value of zero and a standard deviation that can be calculated. For this end we need the sensitivity matrix, S, which, using the compact notation of (5), is given by

$$
S=\frac{\partial a}{\partial p}, \quad \text { or } S_{i j}=\frac{\partial a_{i}}{\partial p_{j}} .
$$


Each column of this matrix is the change in the signal due to a unit change in the corresponding parameter value. In [19] it is shown that the covariance matrix for the errors in the unknown parameters is given by

$$
C=\sigma^{2}\left(S^{T} S\right)^{-1} .
$$

The standard deviation for the $\mathrm{i}^{\text {th }}$ parameter is given by

$$
\sigma_{i}=\sqrt{C_{i i}} .
$$

The results of these calculations for some specific situations are given in the next section.

\subsection{Prediction of attainable position resolution in $r$ for single interactions}

The theory of the last section was used to predict the attainable resolution in the $r$ variable for the detector used in our experiments. Noise-free signals were predicted for our detector by Kai Vetter at Lawrence Berkeley National Laboratory. The predictions were normalized to correspond to a single interaction of $1 \mathrm{MeV}$. Electronic noise was taken into account using a noise model that was $20 \%$ smaller (rms) than that shown in figure 4 (based on measurements obtained with a previous version of the system). Figure 5 shows the resulting rms error for the position resolution in $\mathrm{mm}$ as a function of $r$ and $z$. The resolution is inversely proportional to the interaction energy, so the error for a 100-keV interaction would be ten times larger. If the noise levels shown in figure 4 had been used in the calculation, the results would be $20 \%$ larger.

Note that the attainable radial position resolution is degraded in the noncoaxial region as one would anticipate. There is also a region of somewhat degraded radial position resolution in the portion of the coaxial region where the 
drift times of the electrons and holes are approximately equal. While these results take into account the effects of the variations in expected signal shapes and electronic noise, they do not include the effects of finite electron range. (For electron energies greater than about $250 \mathrm{keV}$ the mean range in germanium is greater than the predicted achievable radial position resolution.) Various sources of possible systematic error, such as unknown detector non-uniformities, would also degrade the position resolution. Nevertheless, these results are very encouraging. They indicate that with care one should be able to obtain radial position resolutions better than $1 \mathrm{~mm}$ over a large volume of the detector for the energy range of interest.

\subsection{Compton suppression via pulse shape analysis}

The suppression of Compton background in $\gamma$-ray spectra is usually performed by surrounding the primary detector (e.g., a Ge crystal) with a secondary detector (e.g., BGO annulus) operated in anti-coincidence mode. Unfortunately, the large size, weight, and cost of such a system can offset expected benefits, especially for measurements performed in the field. For this reason, a Compton suppression technique based only on details of the charge pulse shape seems attractive.

Recent efforts to apply Ge detector pulse shape analysis (PSA) to Compton suppression [7-11] have focused on general characteristics of the charge pulse shape (rise-time, single-site vs. multiple-site shape, etc.). In this project we developed and tested a more fundamental approach based on a complete unfolding of the charge pulse shape into discrete components associated with individual $\gamma$-ray interactions. This approach allows the 
application of an algorithm that favorably rejects Compton escape events. Our algorithm was chosen to allow for discrimination on both single-site and multiple-site escape events. This differs from the algorithms of [10-11] that were designed to reject only single-site events. Below and in [20] we present the details of our algorithm along with experimental results from a real-time implementation on a $5 \mathrm{~cm} \times 5 \mathrm{~cm}$ HPGE detector.

The approach used for Compton suppression was to estimate what fraction of the energy deposited in the detector by a photon was deposited in a single interaction. If this fraction exceeds a certain value (which depends on the total deposited energy), the signal is assumed to be an escape event rather than a full-energy event. Using the least-squares fitting method of Section 2.2 we can determine the number of interactions and the energy and location of each interaction. We could then take the ratio of the energy of the largest interaction to the sums of the energies of all interactions and derive our fraction. Unfortunately, we don't know how to solve equation (8) rapidly enough to get the required throughput (thousands of photons per second). To obtain the required speed, we replace the non-linear least squares with a linear leastsquares approximation.

\subsection{Calculation of zone vector from data}

Equation (8), which comes from equation (4), is non-linear in the parameters $t_{0}$ and $r_{i}$, but is linear in the parameters $E_{k}$. We remove the parameter, $t_{0}$, from the fitting calculations by estimating a value for it, then leaving the value fixed during the fitting. The time of the interaction, $t_{0}$, is estimated by digitally simulating amplitude and rise time compensated (ARC) timing. 
The parameters, $r_{j}$, are removed from the fitting by assuming a fixed list of values for $r_{j}$. For values of $r_{j}$ in this list for which the calculated energy is near 0 , it is assumed that no interaction took place. The values for $r_{j}$ are chosen by dividing the detector into $N$ radial zones of equal "width" (see figure 6). The values for $r_{j}$ are at the centers of the zones. We typically use $N=10$, but shall keep it general for this discussion. Equation (4) now becomes

$$
y_{j}=S\left(t_{j}\right)=\sum_{i=1}^{N} E_{i} K\left(t_{j}-t_{0}, r_{i}\right)+e_{j},
$$

where the sum is now over the number of zones rather than the number of interactions, and the only unknowns are $E_{i}$. If the energy deposited in each zone were deposited at the center, then $E_{i}$ would be the energy in the $\mathrm{i}^{\text {th }}$ zone. We call the (column) vector made up of the $N$ values of $E_{i}$ the zone vector.

The zone vector is calculated as the solution to equation (13) which has non-negative values for all of the $E_{i}$ and which minimizes the sums of the squares of the $e_{j}$. If we do not apply the constraint that all of the $E_{I}$ have non-negative values, we call the resulting zone vector the unconstrained solution. When the constraint is applied we call the result the constrained solution. With perfect data we would obtain a few positive values for $E_{i}$ (those for the zones in which energy was deposited) and zeros for the other values. In the presence of noise, some of the values of $E_{i}$ that should be zero are positive and some are negative. Applying the constraint reduces the effect of the noise on all of the components of the zone vector-even those for which $E_{i}>0$.

The first step in calculating the constrained solution is to calculate the unconstrained solution. If we let $y$ be the column vector with components $y_{\mathrm{j}}$ (the measured signal values), $E$ be the zone vector, and $\mathbf{K}$ be the matrix with 


$$
K_{j i}=K\left(t_{j}-t_{0}, r_{i}\right)
$$

then the zone vector is given by the least squares solution of the matrix-vector equation

$$
y=\mathbf{K} E \text {. }
$$

The solution to this equation is given by

$$
E=\left(\mathbf{K}^{\mathrm{T}} \mathbf{K}\right)^{-1} \mathbf{K}^{\mathrm{T}} \mathbf{y}=\mathbf{U} \boldsymbol{y} .
$$

The matrix, $\mathbf{U}=\left(\mathbf{K}^{\mathrm{T}} \mathbf{K}\right)^{-1} \mathbf{K}^{\mathrm{T}}$, needs only to be calculated once for the detector. It is called the pseudo-inverse of $\mathbf{K}$. The unconstrained solution is the first estimate in an iterative solution, by the method of steepest descent, for the constrained solution.

The method of steepest descent is not a particularly good method for solving this problem. We use it because we can get an acceptable solution very rapidly. Individual iterations can be calculated quickly, but convergence can be slow. We calculate five iterations and accept the answer.

We performed simulations to determine if the added accuracy of the constrained solution is necessary. We simulated $500 \mathrm{keV}$ interactions at the center of one zone, added noise (representative of our detector) to the data and looked at the standard deviation in the calculated energy in this zone. With the unconstrained solutions the standard deviation was $8.3 \%$ of the energy, with the constrained solution it was reduced to $3.4 \%$. This improvement is important for Compton suppression performance. 


\subsection{Zone Leakage}

Equation (13) gives an accurate representation of the how much energy was deposited in each zone only if energy is deposited at the center of the zone. If a single interaction occurs at the boundary between two zones, then (to a first approximation) half of the energy will be calculated to be in each zone. This error is referred to as zone leakage. As the point of interaction is moved from the center of one zone to the center of an adjacent zone the calculated distribution of energy between the two zones varies nearly linearly with the interaction position, as can be seen from figure 7 .

The Compton suppression algorithm tests whether or not a certain fraction of the total energy (usually $\geq 70 \%$ ) is deposited in a single zone. Zone leakage severely hampers this test, because it spreads energy across two zones. To eliminate this problem, we test whether or not the sum of any two energies in adjacent zones exceeds the specified fraction.

\subsection{Optimized zone width}

The proper test for an escape event is based on the fraction of energy deposited in a single interaction, not in a single zone. We use zones only to speed up the calculations. If the zone width were small enough that there is negligible probability that two interactions occur in the same zone, the two tests would be the same. Thus, making the zone width as small as possible reduces errors due to multiple interactions in the same zone. However, as the zone width gets smaller, the matrix, $\mathbf{K}$, in equation (14) becomes closer to being singular. This means that the values in the matrix, $\mathbf{U}$, of equation (15) get large-causing the noise to have more influence on the results. 
We determined the optimum zone width by modeling. Monte Carlo simulations of ${ }^{137} \mathrm{Cs}$ with a high ${ }^{60} \mathrm{Co}$ background were used to obtain a set of interaction positions and energies for a large number of incident $\gamma$-rays. We then constructed a zone vector for each $\gamma$-ray event (ignoring zone leakage) from this data by separating the energy of each interaction into one of a number of radial position zones. This was done using 5, 6, .., 20 zones. We then calculated the effectiveness, defined in (16) in the next section, for each of the zone configurations using an algorithm similar to that described in the next section. We observed the highest effectiveness using 10 zones.

\subsection{Performance of real-time Compton suppression algorithm}

Using the zone vector as obtained above, one can then determine $\mathrm{E}^{*}$, the maximum fraction of the total energy $\left(E_{\text {dep }}\right)$ which is deposited in a single radial zone. This value is then input into a Compton suppression algorithm that makes the "accept-or-reject" decision for the event. The details of this algorithm have been discussed in [20]. The fundamental principle is that a full energy event (above a few hundred $\mathrm{keV}$ ) will usually involve several interaction sites, thus giving a low $\mathrm{E}^{*}$. A Compton escape event, on the other hand, will typically involve only one interaction site, and thus will give a higher $\mathrm{E}^{*}$ (in principle, $\left.E^{*}=1.0\right)$. This allows some degree of discrimination between full energy events (which we want) and Compton escape events (which we do not want).

In [20], the performance of the algorithm was tested using a Europium-152 source, and the results were compared to the performance of a standard BGO suppression system. The figure-of-merit for the comparison was the 
effectiveness $(\varepsilon)$, which is equal to the square root of the counting time improvement. In a high background environment, the effectiveness takes the following form:

$$
\varepsilon=\frac{\mathrm{P}^{\prime}}{\mathrm{P}} \sqrt{\frac{\mathrm{B}}{\mathrm{B}^{\prime}}},
$$

where $B\left(B^{\prime}\right)$ is the background under the peak of interest in the unsuppressed (suppressed) spectrum, and $\mathrm{P}\left(\mathrm{P}^{\prime}\right)$ is the peak height, after background subtraction, in the unsuppressed (suppressed) spectrum.

It was seen in [20] that although modest reductions in counting time could be realized using the current algorithm, the performance was still somewhat below that of a BGO suppression system. However, the pulse shape suppression system wins, by a large margin, in the areas of cost, weight and size. In this respect, it is possible that some field applications might favor the current method.

As discussed in [20], the algorithm rejects those events with $E^{*}$ below the Compton edge for $E_{\text {dep }}$. This rejects those events with $E^{*}=1$, as well as some additional events with $E^{*}<1$. This means that, in principle, we can reject multiple-site Compton escape events as well as the $E^{*}=1$ single-site events. However, in practice, this is not always true. The more interaction sites an event has, the more it resembles a full absorption event. As such, it is found that the algorithm works best when it is truly discriminating a single-site Compton escape event from a multiple-site full energy event. This condition is best realized when one is measuring $\varepsilon$ for a small peak sitting atop a large Compton edge from a higher energy line. Figure 8 shows such a case, which is the 1112 $\mathrm{keV}$ peak in Eu-152 sitting atop the $1118 \mathrm{keV}$ Compton edge from the $1332 \mathrm{keV}$ 
line in Co-60. In this case, the source was placed on the side of the detector (rather than in front, on-axis), and the quasi-planar region was blocked with a lead brick. As discussed below, by blocking out the quasi-planar region we can maximize the effectiveness. Using this configuration, we obtain a value of $\varepsilon=1.52$ \pm 0.09 , which is better than the values presented in [20]. For peaks that sit atop a high Compton background, but not necessarily the Compton edge, one can still expect a significant increase in performance. For example, for the set-up just described, the $778 \mathrm{keV}$ line from Eu-152 (which does not sit atop a Compton edge) gives a value of $\varepsilon=1.35 \pm 0.05$, which is also higher than the value presented in [20].

It had been speculated in [20] that possible improvements to the Compton suppression algorithm could be realized by artificially raising or lowering the cutoff curve. Tests that have been done so far have not verified this. In particular, if one raises the cutoff curve by $10 \%$, the effectiveness for the $1112 \mathrm{keV}$ line described above is the same within error. If one lowers the cutoff curve by $10 \%$, the effectiveness is reduced to $1.20 \pm 0.06$.

The poor performance of the algorithm in the quasi-planar region is due to the different shape of the internal detector electric field in that region. Since the basis functions used to fit the charge pulse shape are based only on the coaxial part of the detector, the pulse shapes in the quasi-planar region are not well fit, and thus the algorithm will not work well there. Figure 9 shows a scan of our HPGe detector from the side using a Eu-152 source (in the presence of another strong Co-60 source). The collimator-hole was $3 \mathrm{~mm}$ in diameter. The cylindrical Ge crystal is $5 \mathrm{~cm}$ long by $5 \mathrm{~cm}$ wide. The effectiveness results show 
that the warped electric field in the front region strongly affects performance there, but the rest of the crystal displays fairly uniform performance.

Figure 10 shows a scan of the detector from the front face using the same set-up as above. The results show that the performance of the algorithm varies inversely with radial position. This is probably due to the fact that multiple site escapes (which are hard to reject) occur more often for $\gamma$-rays which first interact near the edge of the detector.

Another factor that is known to affect charge pulse shapes is the directional dependence of the charge carrier drift velocity with respect to the crystallographic axes [21]. For standard Ge detectors, the bore axis is parallel to the [001] crystallographic axis, while the [100] and [010] directions are perpendicular to the coaxial surface. This means that for charge drifting in the pure coaxial part of the detector, the velocity (and hence the pulse shape) will vary with azimuthal angle. In particular, the drift velocity will be maximum along the $<100>$ axis, and minimum along the $<110>$ axis. This is expected to lead to an oscillating azimuthal dependence of the drift velocity with a period of $90^{\circ}$ and a net variation of $10 \%$ on the magnitude [21].

To investigate the azimuthal dependence of the Compton suppression algorithm, we collimated a beam of $662 \mathrm{keV} \gamma$ rays (from Cs-137) onto the Ge detector front face. The collimator hole in this case was $3 \mathrm{~mm}$ in diameter. Figure 11 (top) shows the seven locations at which data was acquired. The sites chosen sweep out a $90^{\circ}$ arc on the circle at radius $15 \mathrm{~mm}$ (mid-way between the edge of the inner bore and the outside edge). This range should necessarily include both a maximum and minimum in the drift velocity. Figure 11 (bottom) shows the 
results. While some variation is seen, it is less than $10 \%$ peak-to-peak. Further experimental study of the azimuthal dependence of the drift velocity is in progress [22].

\subsection{Gamma-ray imaging via pulse shape analysis}

Standard Compton cameras [23] use two planar Ge strip detectors separated by a large distance. The need to operate these detectors in coincidence can lead to a very low efficiency. For example, two $5 \mathrm{~cm} \times 5 \mathrm{~cm} \times 1 \mathrm{~cm}$ planar Ge strip detectors separated by $10 \mathrm{~cm}$ could be operated as a Compton camera in order to image a $400 \mathrm{keV} \gamma$-ray source located $20 \mathrm{~cm}$ away on axis. However, Monte Carlo simulation [24] shows that the efficiency of such a system is about a factor of 300 less than that of a large volume $(8 \mathrm{~cm} \times 9 \mathrm{~cm})$ HPGe detector. This result indicates that if imaging could be done using a large volume HPGe, orders of magnitude improvement in imaging times could be realized.

A high purity germanium detector can, in principle, be operated as a Compton camera imager by highly segmenting the outer contact and attaching a preamplifier and waveform digitizer to each electrode. For a given $\gamma$-ray event, the set of measured pulse shapes would be used to infer the location of all $\gamma$-ray interaction points. The interaction points could then be time-ordered using $\gamma$-ray tracking, and the first two identified points could be used to do the standard Compton camera imaging. The technique of $\gamma$-ray tracking [25] uses the energyangle relationship of the Compton scattering process to find a sequence of points which is consistent with energy and momentum conservation. In principle, this requires looking at $\mathrm{N}$ ! combinations (assuming $\mathrm{N}$ interaction points). Each 
sequence is assigned a figure-of-merit, and the sequence with the best figure-ofmerit is deemed to be the proper sequence. Once the first two time-ordered points are identified, the energy angle relation of Compton scattering then gives a cone of possible incident directions for the $\gamma$-ray. This cone can be projected onto a virtual image plane. Over many events, these conic sections ("rings") will overlap on the image plane thus producing an image.

The direction of the incident $\gamma$-ray is localized to a cone, not a line, because the information we have about the first Compton scattering is kinematically incomplete. We know the direction of the scattered $\gamma$-ray, $\hat{v}_{1,2}$, from the line connecting the first two scattering points, and we know the polar angle, $\theta_{\gamma}$, relating the incident direction, $\hat{\mathrm{v}}_{\gamma}$, to $\hat{\mathrm{v}}_{1,2}$ (by using the energy-angle relationship), but we do not know the direction of the recoil electron. This gives $\hat{v}_{\gamma}$ an azimuthal uncertainty with respect to $\hat{v}_{1,2}$, and thus generates a cone of possible incident directions. Using a covariance analysis, we have shown that in general it is not possible to determine the direction of the recoil electron in HPGe detectors in the energy range of interest.

\subsection{Prediction of position resolution in $\mathrm{r}, \mathrm{phi}$, and $\mathrm{z}$ for two interactions}

Since position resolution for multiple $\gamma$-ray interactions is very important to $\gamma$-ray imaging, we now discuss this aspect in detail.

In section 2.2 we covered how to estimate the positions and energies of individual interactions from the observed detector signal. This did not explicitly deal with segmented detectors, but extending the analysis to them is straightforward. In equations (3), (4) and (5) we use a vector, $\boldsymbol{y}$, whose 
components are the sampled values of the measured signal. For a segmented detector use the concatenation of the sampled signals for all of the segments for $y$. All of the rest of the analysis is the same.

The resolution calculations require knowledge of the signal shapes on each detector segment. These were calculated with certain assumptions. It was assumed that the charge carriers moved at constant (saturated) velocity in the radial direction. This means that the calculations are only valid away from the ends of the detector. End effects cause an axial component of the electric field, which would change the direction of the charge carrier velocity. The radial velocity assumption also requires that the charge density gradient in the detector is small enough that no significant axial field is produced. With these assumptions, the signal shapes were calculated using conventional techniques [26], which require numerically obtaining solutions to Laplace's equation with potentials equal to one on one of the segments and equal to zero on the others.

The spatial resolution depends on the geometry of the detector and its segmentation, on the number of interactions and on the energies and positions of the individual interactions (four parameters per interaction). We assumed a coaxial detector of the same dimensions as the detector we used for the Compton suppression experiments. The segmentation was two taken as uniform along the axial and azimuthal directions. We used interactions, one depositing $156 \mathrm{keV}$ and one depositing $30 \mathrm{keV}$. This combination is of particular interest in detecting highly enriched uranium.

A major factor effecting the resolution with two or more interactions is the spatial separation between the two interactions. As the two interactions get close together, the matrix, $\mathbf{K}$, in (14) rapidly approaches a singular matrix. This 
represents the fact that we can't tell two nearby interactions from one that is between them. For this reason, each simulation was run with a nearly constant separation between the particles.

Once we fix our attention on two interactions with fixed separation, the errors in the spatial coordinates still depend quite strongly on locations, relative to the center of a segment, of the interactions. Interactions near segment boundaries have much less position error than those near the center of the segment. Because of the large number of variables, six coordinates for each interaction pair, the resolution was examined statistically. A large collection of interaction pairs was generated with a fixed separation, and a histogram was made of the calculated error estimates in each coordinate. We display the histogram and its integral. One can read the various percentile points directly from the histogram integral. We quote the $90^{\text {th }}$ percentile error level as the resolution.

Table 1 shows the results for three different situations. The center row is the reference situation with both the $\varnothing$ and the $z$-axes divided into six segments and with separation between interactions of $1 \mathrm{~cm}$. The segments are $1 \mathrm{~cm}$ long in the $\mathrm{z}$ direction and $2.6 \mathrm{~cm}$ long in the $\varnothing$ direction. Note that the resolution is much better than the segment size and that it is roughly inversely proportional to the energy of the interaction.

The top row shows how the resolution degrades with interactions close together. The resolution in $r$ degrades by more than an order of magnitude, and the resolution in $z$ degrades by a factor of three. We don't yet have an intuitive explanation for the extreme difference in the amount of degradation in the different variables. 
The bottom row shows the effect of changing the z-axis segment length to $2 \mathrm{~cm}$. One effect is that all resolutions increase by a factor of the square root of two. This is because of the decrease in signal-to-noise caused by doubling the capacitance of each segment. The z-axis resolution increases by an additional factor of five. For small segment size the resolution will be proportional to size, for large segment size it will be exponential in size.

\subsection{Prediction of the performance of a segmented HPGe detector as a $\gamma$-ray imager}

When generating a $\gamma$-ray image using the segmented HPGe detector concept, there are two factors that will tend to "blur" the picture. The first is the "ring" artifact caused by the projection of the entire probability cone onto the pixelated image plane. The second factor is the blur caused by finite position resolution. This will add an uncertainty to the cone axis, and hence will give a finite "thickness" to the ring (thus increasing the background). In principle, there is also blur caused by the energy uncertainty, which could further increase the ring thickness. However, for Ge detectors, which have very good energy resolution, this component is not significant.

The effect of the blur factors can be seen by viewing a point response function for the imager. Consider a point source placed $10 \mathrm{~cm}$ away (on axis) from a $5 \mathrm{~cm} \times 5 \mathrm{~cm}$ HPGe detector. Figure G12 (black curve) shows a histogram of the image obtained along the $x$-axis of the image plane. This prediction assumes an infinitesimal position resolution, and thus the width of this response is due to the ring artifacts. The angular resolution for this case is about 2 degrees. The red 
line in Figure 12 is a histogram of the same quantity when a 1-mm position resolution is used. The angular resolution here is about 7 degrees.

One can conceive of using a segmented HPGe as a $\gamma$-ray imager in several different scenarios: near field imaging of radionuclide distributions; far field imaging to locate hidden SNM (e.g. checkpoint scenarios at border crossings); or even single photon imaging in astrophysics and elsewhere. Here we simulate the performance of the HPGe $\gamma$-ray imager in each of these scenarios.

First we consider a scenario whereby we image a radioactive ring emitting $1 \mathrm{MeV} \gamma$ rays. The ring is assumed to have an OD of $6 \mathrm{~cm}$ and an ID of $4 \mathrm{~cm}$, and is assumed to sit $10 \mathrm{~cm}$ away (on axis) from a $5 \mathrm{~cm} \times 5 \mathrm{~cm}$ coaxial HPGe detector. Figure 13 shows the evolution of the image as more $\gamma$ rays are collected. While Figure 13 assumes infinitesimal position resolution, Figure 14 shows the image as acquired with a $1 \mathrm{~mm}$ position resolution. For a source of $10 \mu \mathrm{Ci}$, this image would take about 3 minutes to acquire.

Next we consider a checkpoint scenario in which $1 \mathrm{~kg}$ of enriched Uranium is located (perhaps in the back of a truck) 3m from a HPGe detector. The detector itself is sitting $1 \mathrm{~m}$ above the ground. Figure 15 shows the acquired image in false color. This image is for the $186 \mathrm{keV}$ line in Uranium, and assumes a $1 \mathrm{~mm}$ position resolution. The simulated counting time is about 2 minutes.

Figure 16 shows results for a single photon-imaging scenario. With 1-mm position resolution, it seems possible to localize the direction of a single $186 \mathrm{keV} \gamma$ ray to about $4 \%$ of $4 \pi$. 


\subsection{Proof-of-principle experiment at Berkeley}

To demonstrate that a segmented detector can be used for $\gamma$-ray imaging, we are currently in the process of performing tests on an existing segmented Ge detector: the 36-fold segmented GRETA prototype detector at Berkeley [14]. A Compton scatter coincidence experiment has been designed to probe the detector with a specified amount of energy at different internal locations. The set-up [27] consists of a collimated 137Cs source (662 $\mathrm{keV} \gamma$ rays), the GRETA prototype detector (the "primary" detector), 5 slat collimators, and $5 \mathrm{NaI}$ crystals (the "secondary" detectors). We will look for a single 90-degree scatter in the primary detector ( $374 \mathrm{keV}$ deposited) and a full absorption in any of the secondary detectors $(288 \mathrm{keV})$. By varying the position of the front and slat collimators, we can select any position inside the primary Ge crystal. Using this technique, we hope to demonstrate that one can obtain at least a $1 \mathrm{~mm}$ internal position resolution (at $\mathrm{E}=374 \mathrm{keV}$ ) by means of pulse shape analysis.

Using the GRETA prototype detector, we also plan to do an imaging proof-of-principle whereby data would be acquired for an arbitrary source distribution. We would then attempt to image the source distribution using the measured pulse shape sets.

\section{Summary}

In this report we have discussed two applications for digital pulse shape analysis in Ge detectors: Compton suppression and $\gamma$-ray imaging. The Compton suppression aspect has been thoroughly studied during the past few years, and a real-time, laboratory-prototype system has been fielded. A 
summary of results from that set up have been discussed here. The $\gamma$-ray imaging aspect, while not yet developed experimentally, looks very promising theoretically as the simulations presented here have shown. Experimental work currently underway at Berkeley (as discussed in section 4.3) should help further guide us towards the proper developmental path.

Reference herein to any specific commercial product, process, or service by trade name, trademark, manufacturer, or otherwise, does not necessarily constitute or imply its endorsement, recommendation, or favoring by the United States Government or the University of California. The views and opinions of authors expressed herein do not necessarily state or reflect those of the United States Government or the University of California, and shall not be used for advertising or product endorsement purposes.

\section{References}

[1] T.W. Raudorf, Mo.O. Bedwell, T.J. Paulus, IEEE Trans. Nucl. Sci. NS-29 (1982) 764.

[2] G. Knoll, Radiation Detection and Measurement, 2nd edition, (John Wiley and Sons, 1989), p. 402.

[3] G.J. Bamford, A.C. Rester and R.L. Coldwell, IEEE Trans. Nucl. Sci. NS-39 (1992) 595.

[4] J. Roth, J.H. Primbsch and R.P. Lin, IEEE Trans. Nucl. Sci. NS-31 (1984) 367.

[5] P.T. Feffer, D.M. Smith, R.D. Campbell, J.H. Primbsch, P.N. Luke, N.W. Madden, R.H. Pehl and J.L. Matteson, SPIE 1159, EUV, X-Ray and Gamma-Ray Instrumentation for Astronomy and Atomic Physics (1989) p. 287.

[6] F. Petry, A. Piepke, H. Strecker, H.V. Klapdor-Kleingrothaus, A. Balysh, S.T. Belyaev, A. Demehin, A. Gurov, I. Kontratenko, D. Kotel'nikov, V.I. Lebedev, D. Landis, N. Madden and R.H. Pehl, Nucl. Instr. and Meth., A 332 (1993) 107.

[7] A. Del Zoppo, C. Agodi, R. Alba, G. Bellia, R. Coniglione, K. Loukachine, C. Maiolino, E. Migneco, P. Piatelli, D. Santonocito and P. Sapienza, Nucl. Instr. and Meth., A334 (1993) 450.

[8] B. Aspacher and A.C. Rester, Nucl. Instr. and Meth., A338 (1994) 511.

[9] B. Aspacher and A.C. Rester, Nucl. Instr. and Meth., A338 (1994) 516.

[10] B. Philhour, S.E. Boggs, J.H. Primbsch, et al., Nucl. Instr. and Meth., A403 (1998) 136.

[11] F. Petry, Prog. Part. Nucl. Phys. 32 (1994) 281. 
[12] Th. Kröll, I. Peter, Th.W. Elze, J. Gerl, Th. Happ, M. Kaspar, H. Schaffner, S. Schremmer, R. Schubert, K. Vetter, H.J. Wollersheim, Nucl. Instr. and Meth., A371 (1996) 489.

[13] J.J. Friel and R.B. Mott, Advanced Materials and Processes, 145 (Feb. 1994) 35.

[14] M.A. Deleplanque, I.Y. Lee, K. Vetter, G.J. Schmid, F.S. Stephens, R.M. Clark, R.M. Diamond, P. Fallon, and A.O. Macchiavelli, submitted to Nucl. Instr. and Meth., 1998, (LBNL-42443 Preprint).

[15] A. M. Rosie, Information and Communication Theory, $2^{\text {nd }}$ Edition, Van Nostrand Reinhold Company, London, 1973.

[16] Harry L. Van Trees, Detection, Estimation, and Modulation Theory, Part I, John Wiley and Sons, New York, 1968.

[17] W. Press, S. Teukolsky, W. Vetterling and B. Flannery, Numerical Recipes in C--The Art of Scientific Computing. Cambridge University Press, 1992.

[18] W. Davenport and W. Root, An Introduction to the Theory of Random Signals and Noise, IEEE Press, 1987.

[19] J. Blair, D. Beckedahl, J. Kammeraad, G. Schmid, Nucl. Instr. and Meth., A 422 (1999) 331.

[20] G.J. Schmid, D. Beckedahl, J.J. Blair, A. Friensehner, J.E. Kammeraad, Nucl. Instr. and Meth., A 422 (1999) 368.

[21] S.A. Slassi-Sennou, S.E. Boggs, P.T. Feffer, R.P. Lin, The Transparent Universe, Proc. $2^{\text {nd }}$ Integral workshop, ESA SP-382, 627 (1997).

[22] G.J. Schmid et al, Work in progress at LLNL.

[23] B.F. Philips et al., IEEE Trans. Nucl. Sci. 43, 1472 (1996).

[24] R. Brun, et al., GEANT3 users' guide, DD/EE/84-1, CERN (1987).

[25] G.J. Schmid, M.A. Deleplanque, et al., Accepted at NIM A (1999).

[26] Journal of Applied Physics 85, 647 (1999).

[27] Kai Vetter, Lawrence Berkeley Lab, private communication 


\begin{tabular}{|l|c|c|c|c|c|c|}
\hline \multirow{2}{*}{ Energy (keV) } & \multicolumn{3}{|c|}{30} & \multicolumn{3}{c|}{156} \\
\hline Parameter & $\mathrm{r}_{1}$ & $\emptyset_{1}$ & $\mathrm{z}_{1}$ & $\mathrm{r}_{2}$ & $\emptyset_{2}$ & $\mathrm{z}_{2}$ \\
\hline $\begin{array}{l}\text { 6x6, .5 cm point } \\
\text { separation }\end{array}$ & $>5$ & 1.5 & .9 & 1.1 & .30 & .16 \\
\hline $\begin{array}{l}\text { 6x6, 1 cm point } \\
\text { separation }\end{array}$ & .22 & 1.2 & .33 & .08 & .21 & .06 \\
\hline $\begin{array}{l}\text { 6x3, 1 cm point } \\
\text { separation }\end{array}$ & .4 & 1.9 & 2.3 & .08 & .35 & .45 \\
\hline
\end{tabular}

Table $1.90^{\text {th }}$ Percentile error $(\mathrm{mm})$ for three different situations. 


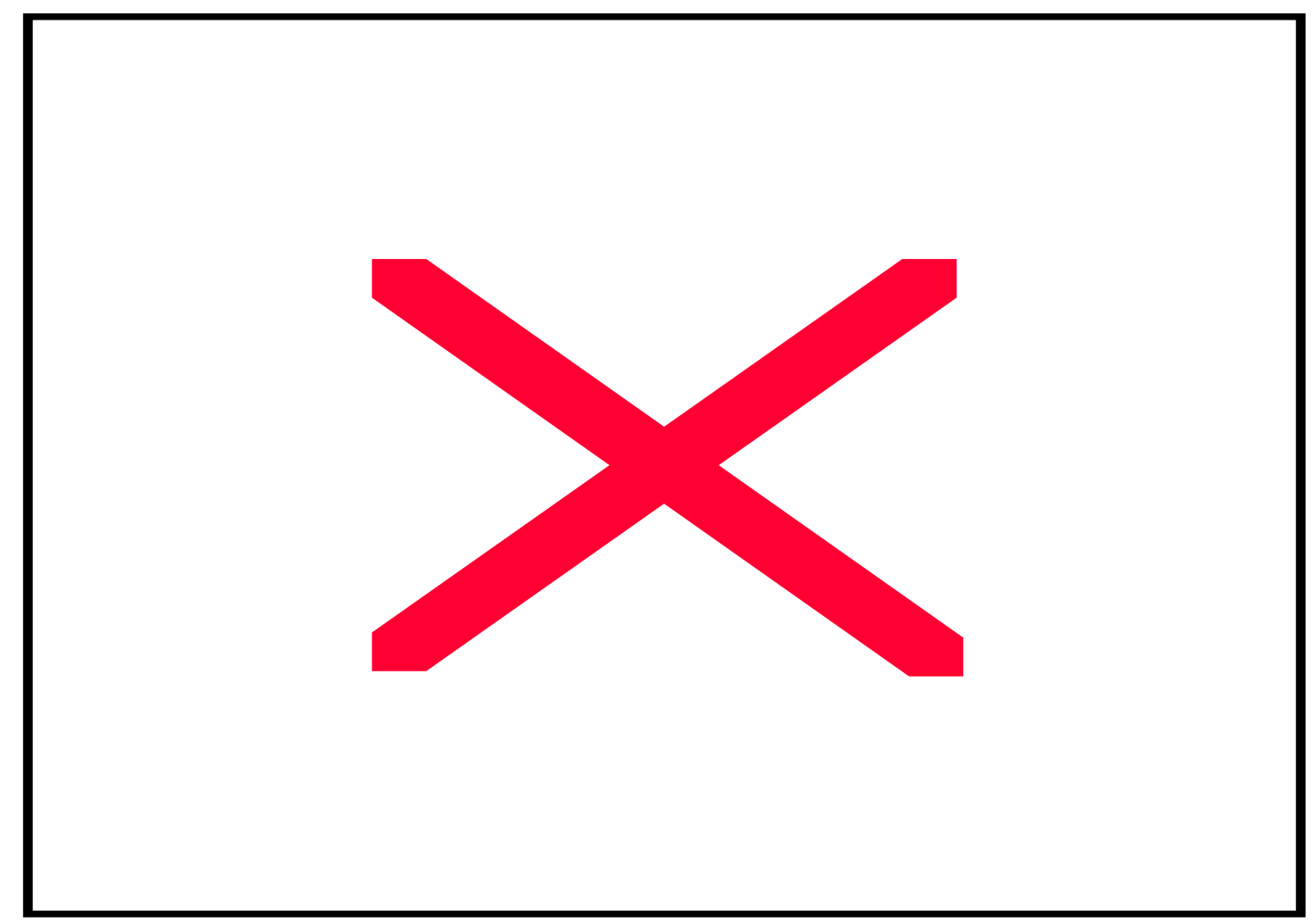

Figure 1: Predicted signals vs. $\mathrm{r}$ in a 5-cm diameter coaxial HPGe detector 


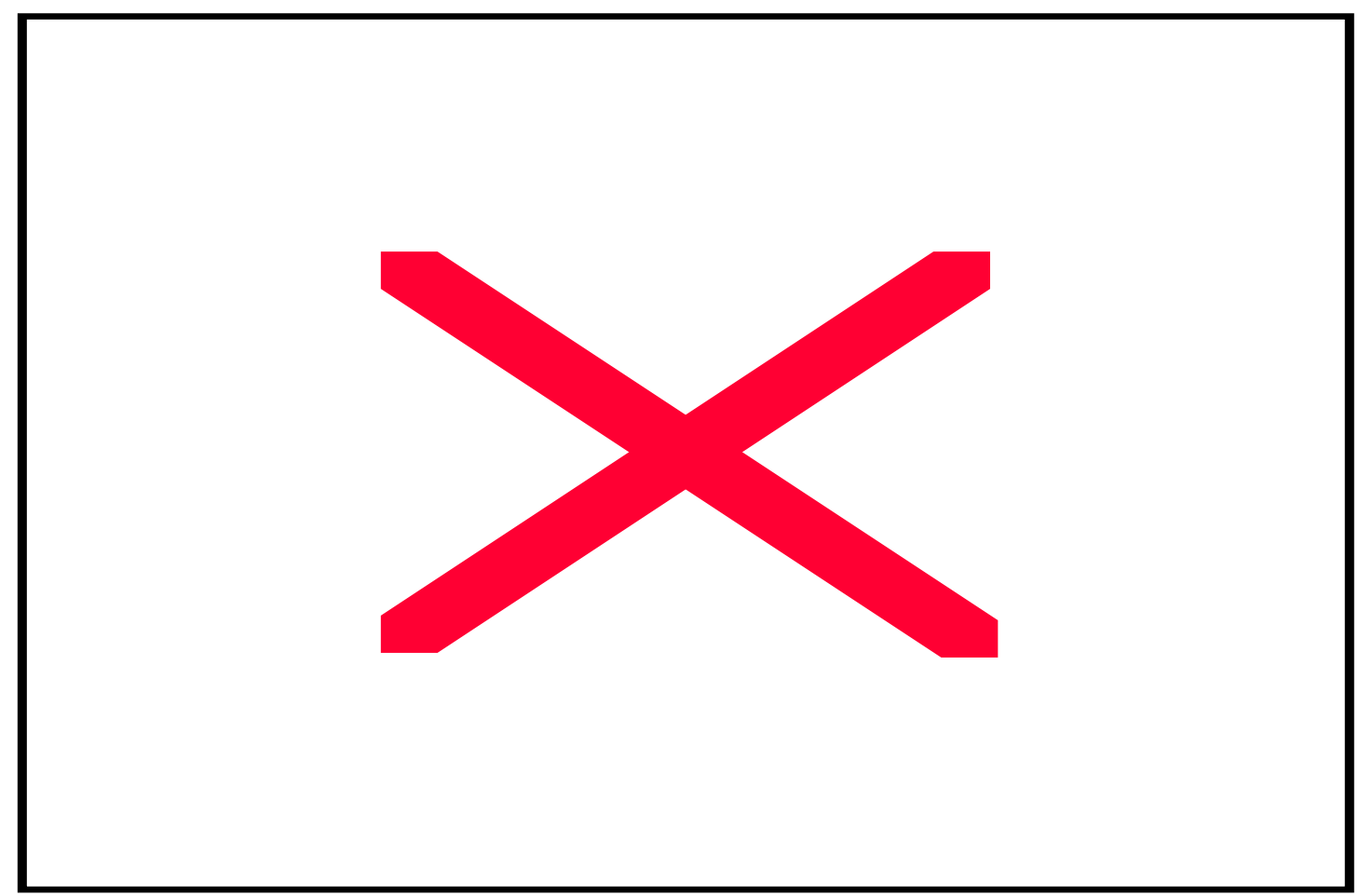

Figure 2: Comparison of measured and predicted radial response functions at $\mathrm{r}=15 \mathrm{~mm}$. 


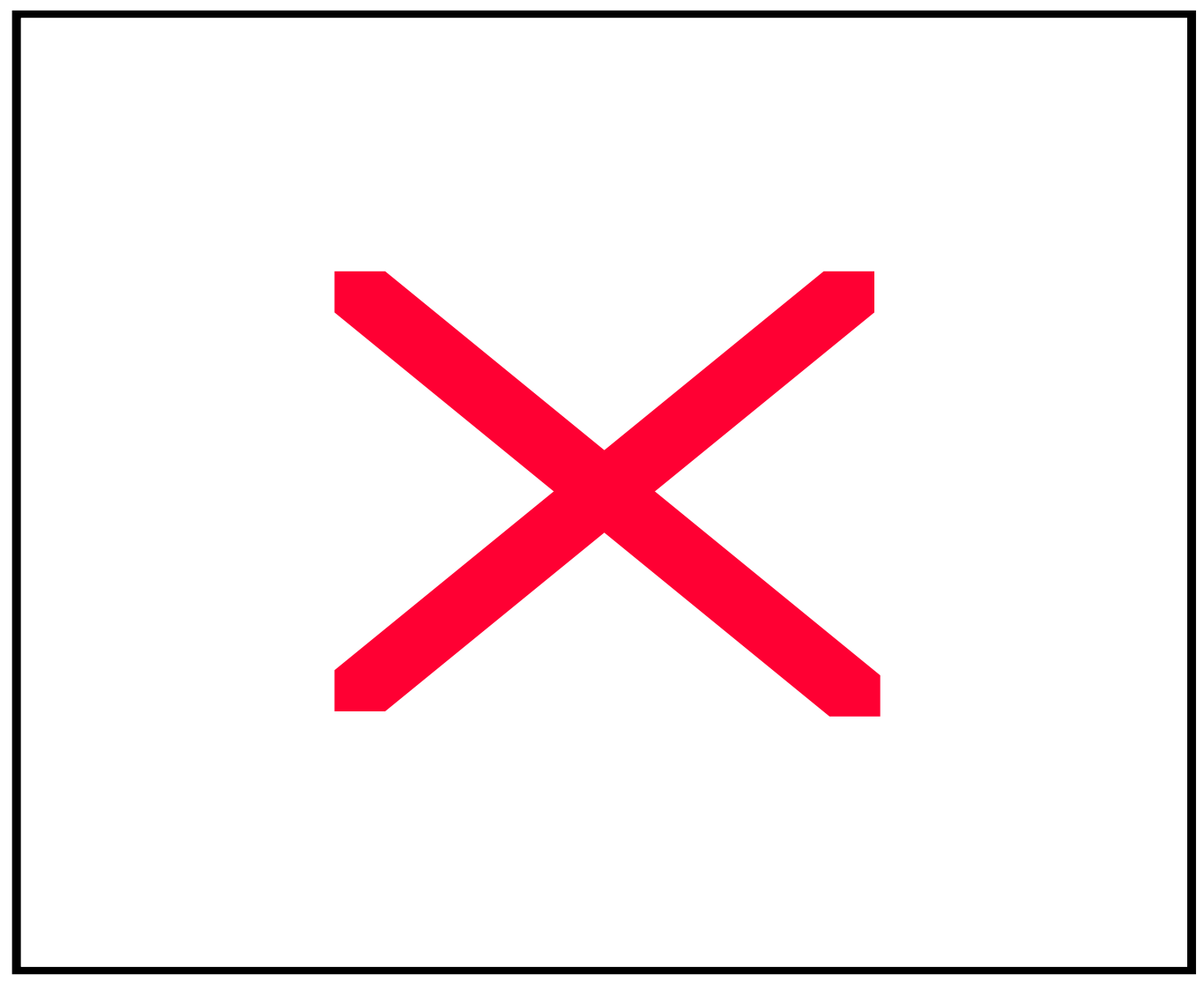

Figure 3: Preamplifier frequency response 


$$
x
$$



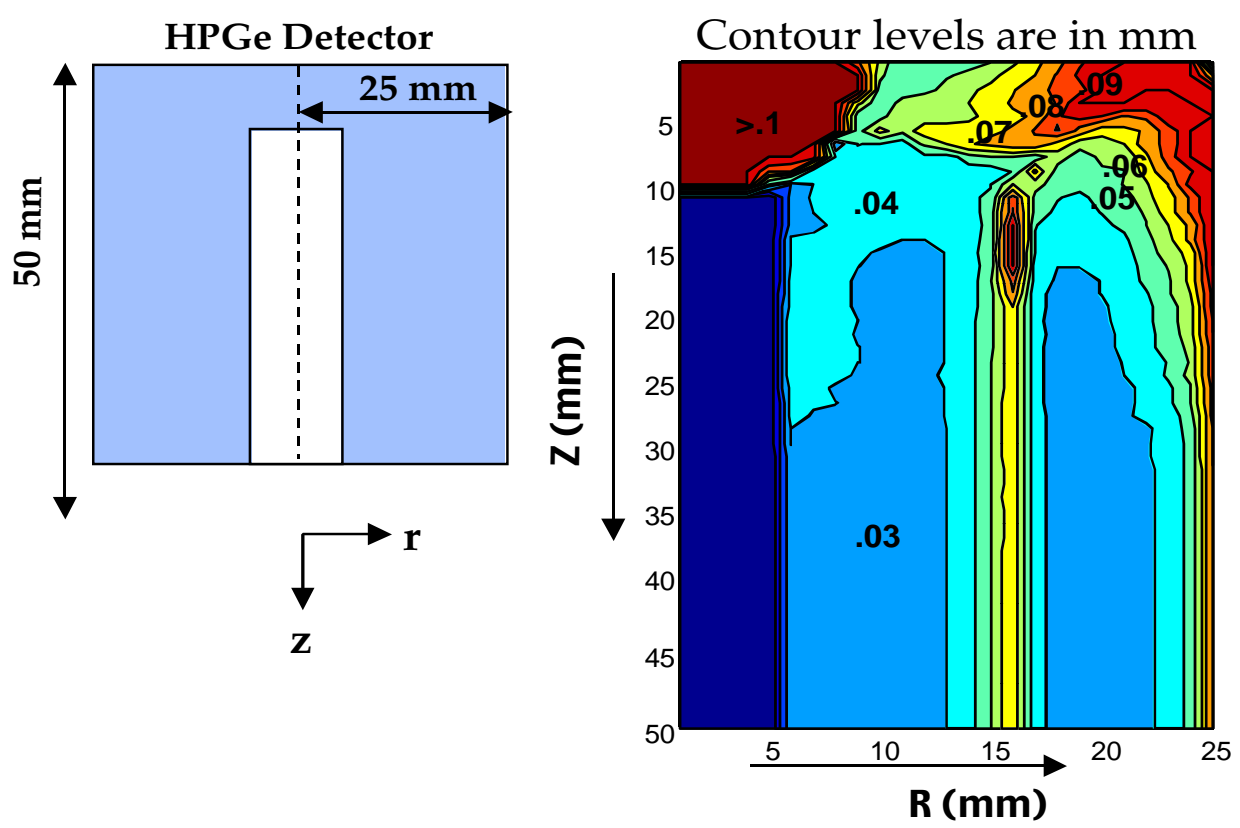

Figure 5: Contour map showing the calculated position resolution for a $1-\mathrm{MeV}$ deposition at one location. The results scale inversely with energy. 

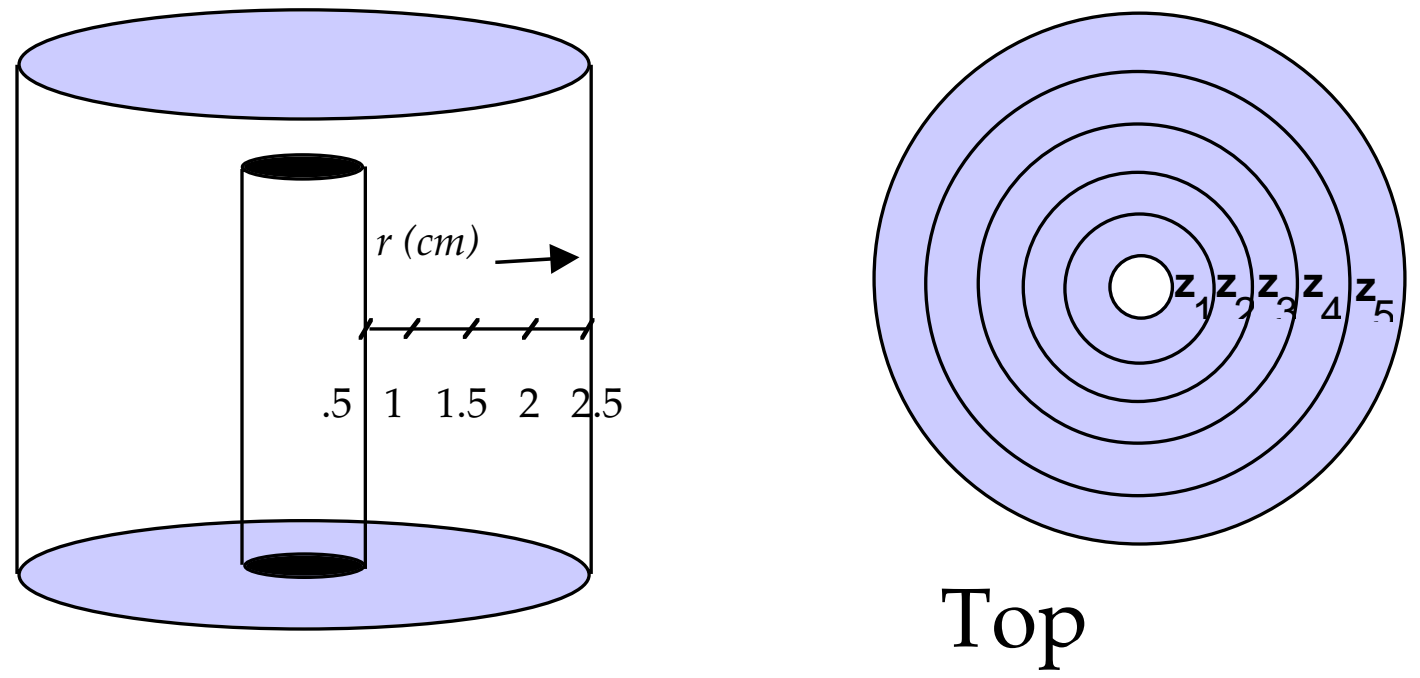

Figure 6: Detector divided into zones 


$$
x
$$




\section{Compton suppression}

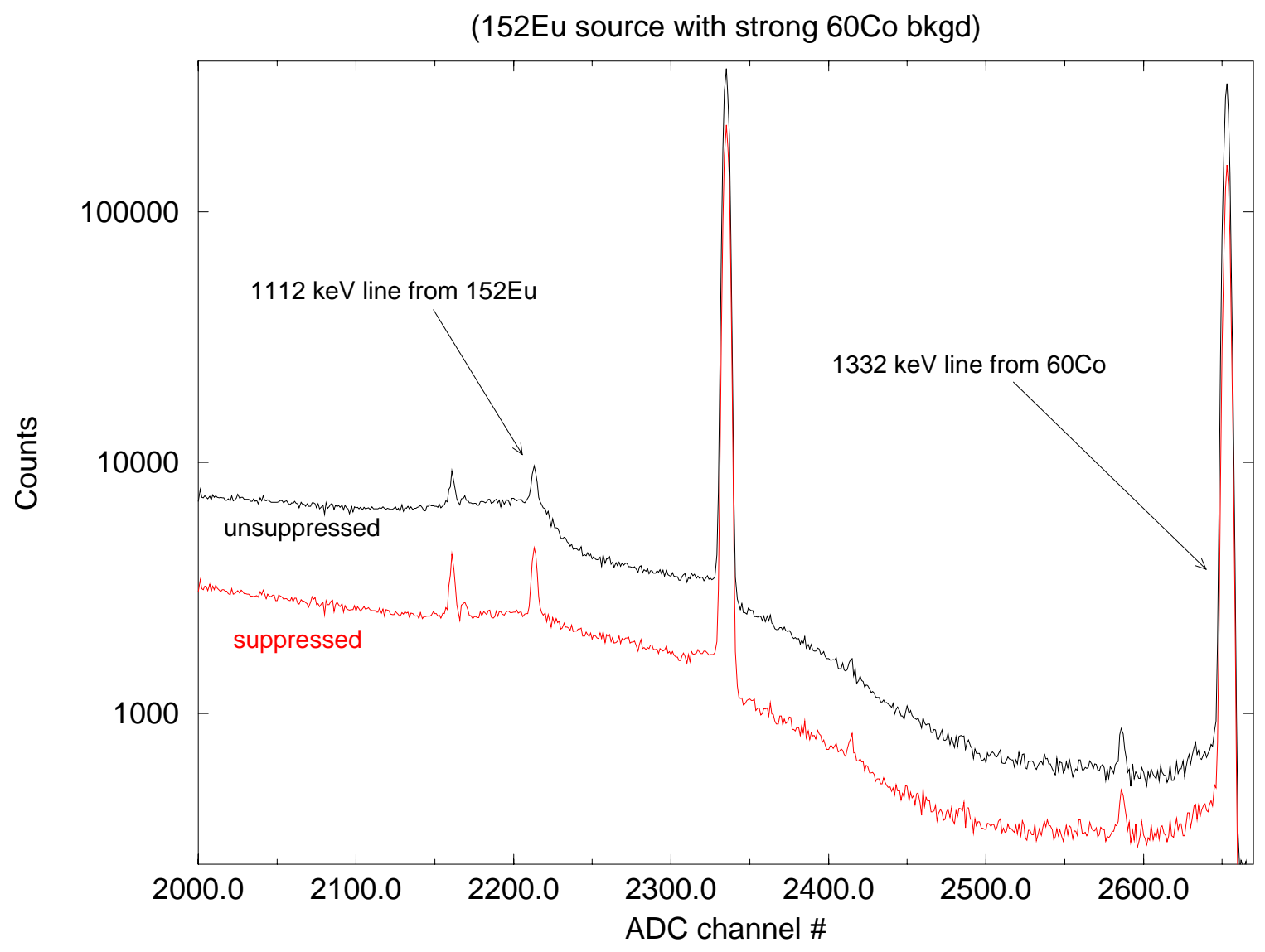

Figure 8: Example of measurement in which the Compton suppression method by pulse shape analysis is most effective. The $1112 \mathrm{keV}$ peak in Eu-152 falls on the $1118 \mathrm{keV}$ Compton edge from the $1332 \mathrm{keV}$ line in Co-60. 
Side scan of $20 \%$ HPGe with 152Eu source

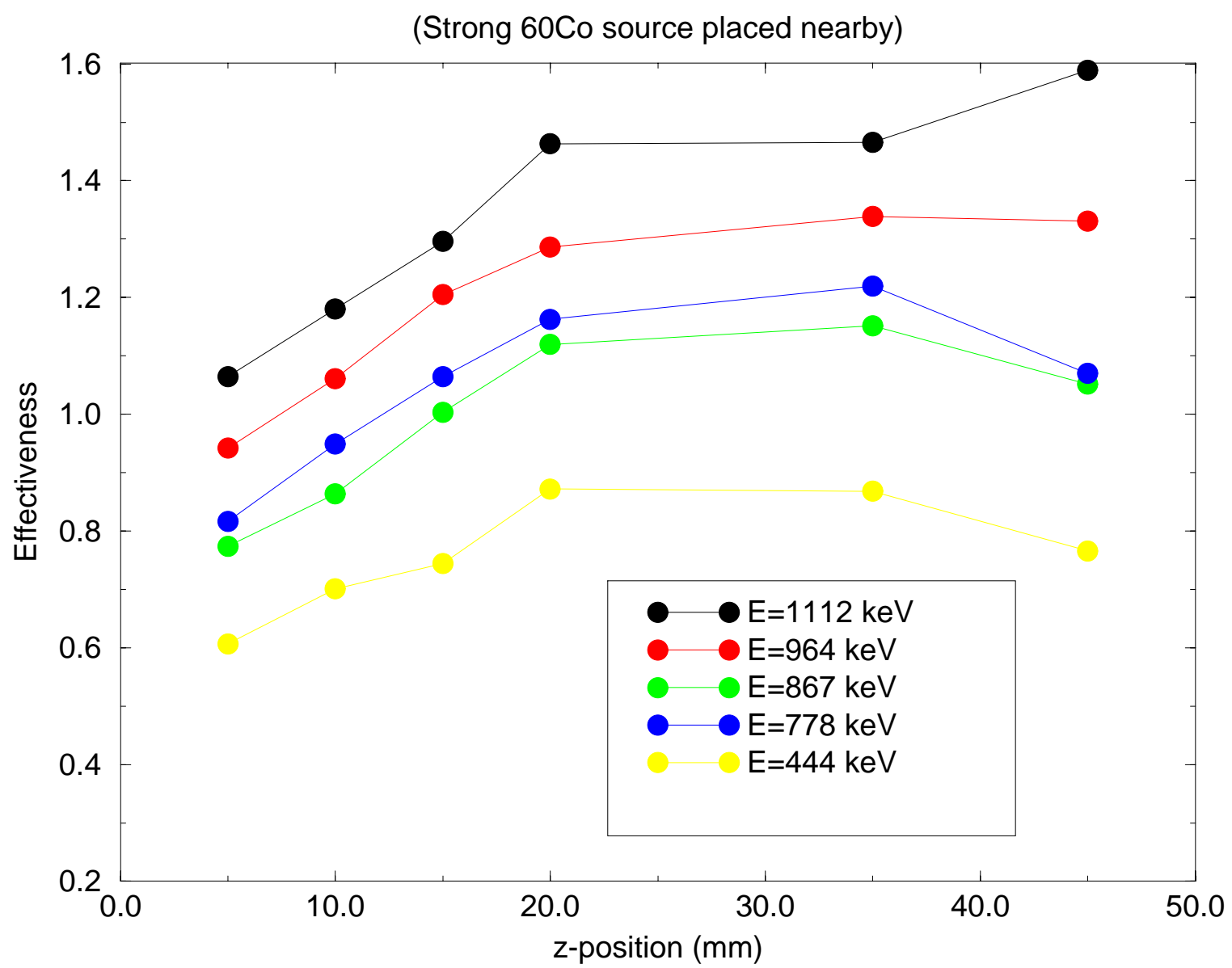

Figure 9: Compton suppression effectiveness versus $\mathrm{z}$ (side scan) for our $5 \mathrm{~cm} \mathrm{x}$ 5cm HPGe detector using a Eu-152 source in the presence of another strong Co60 source. 


\section{Front scan of $20 \%$ HPGe with 152Eu source}

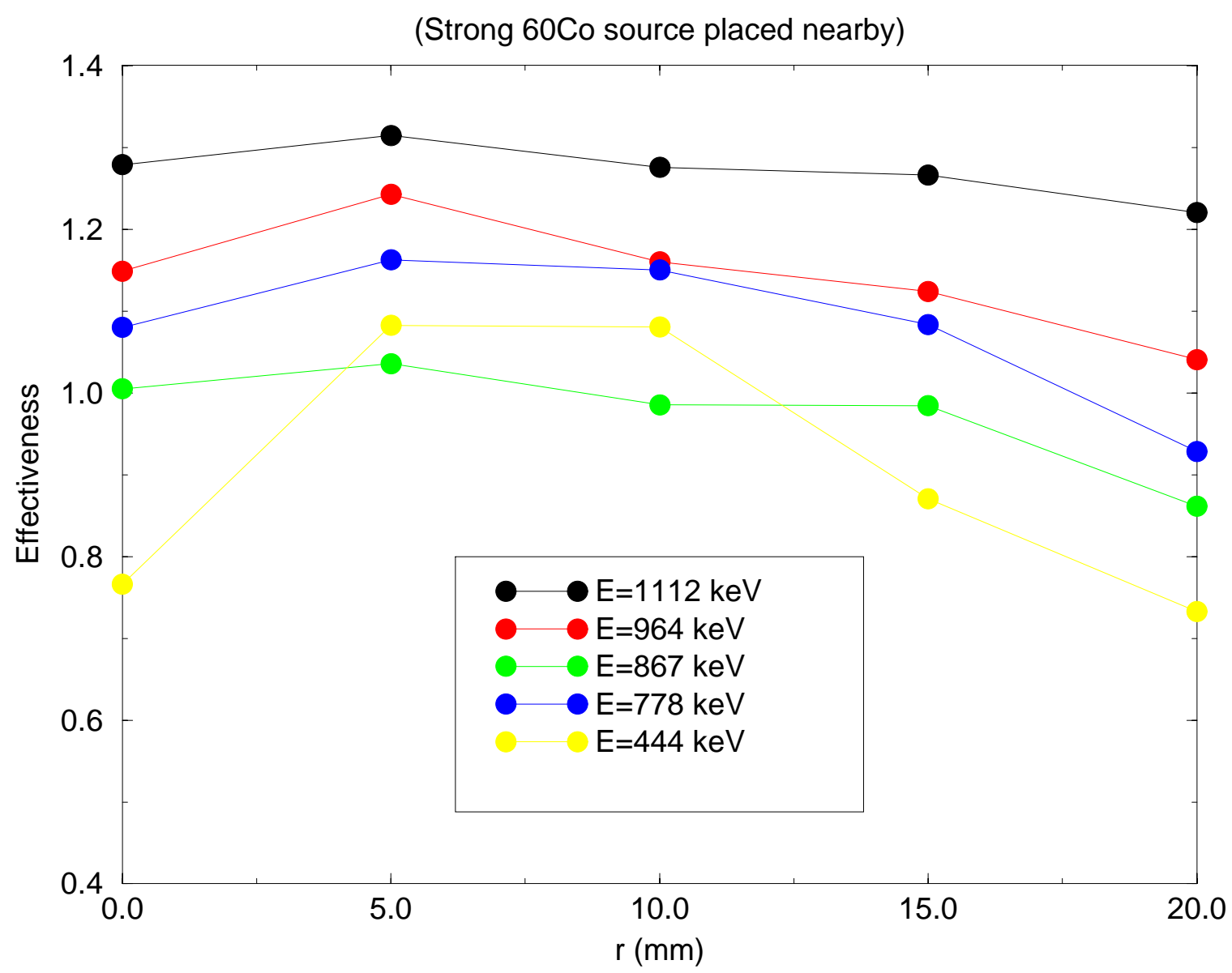

Figure 10: Compton suppression effectiveness versus $r$ (front face scan) for our HPGe detector. 


\section{Compton suppression algorithm}
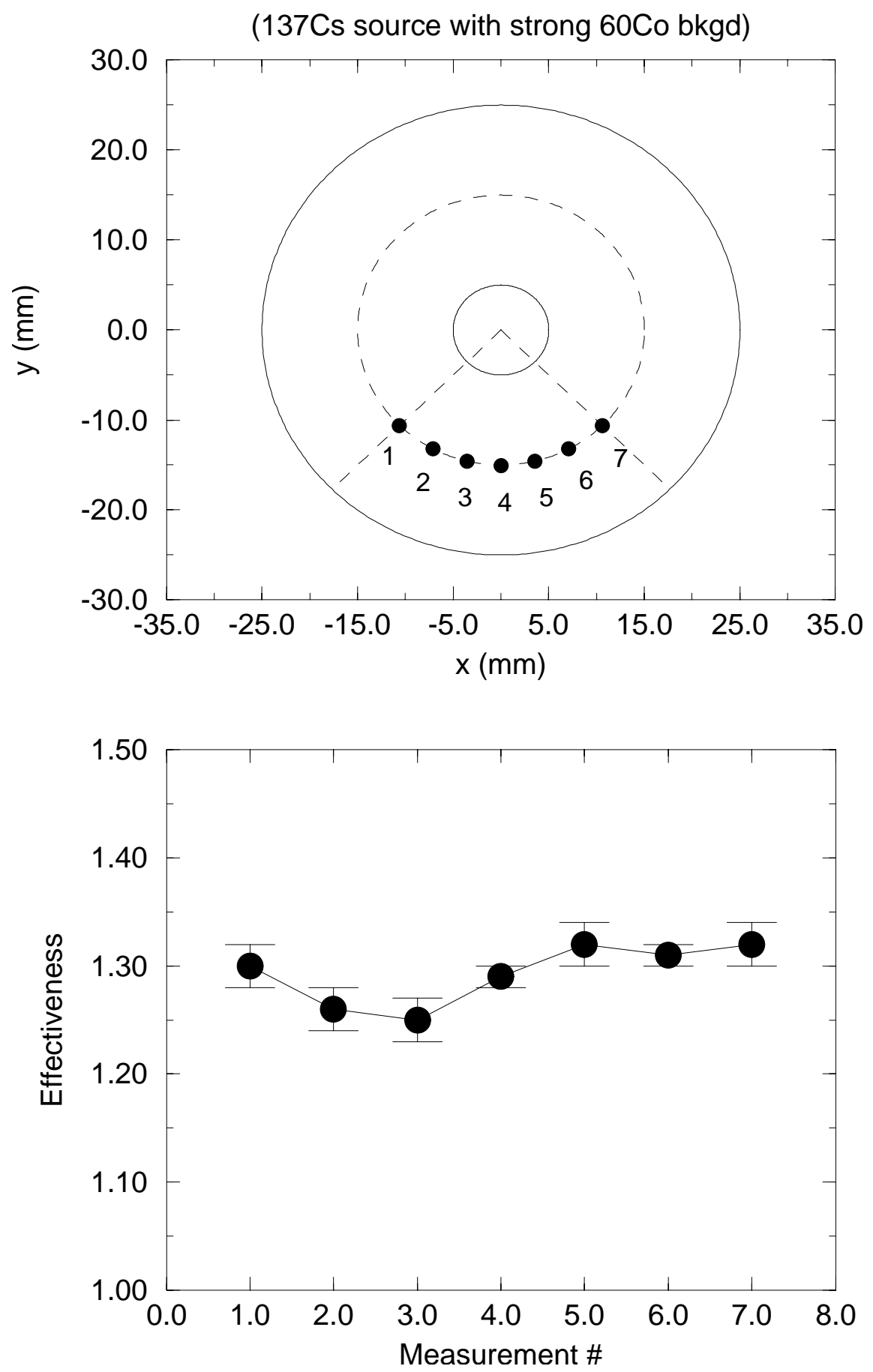

Figure 11: (a) The seven locations at which data was acquired in the azimuthal scan; (b) Compton suppression effectiveness versus azimuth for our HPGe detector. 
Point response function for gamma ray imager

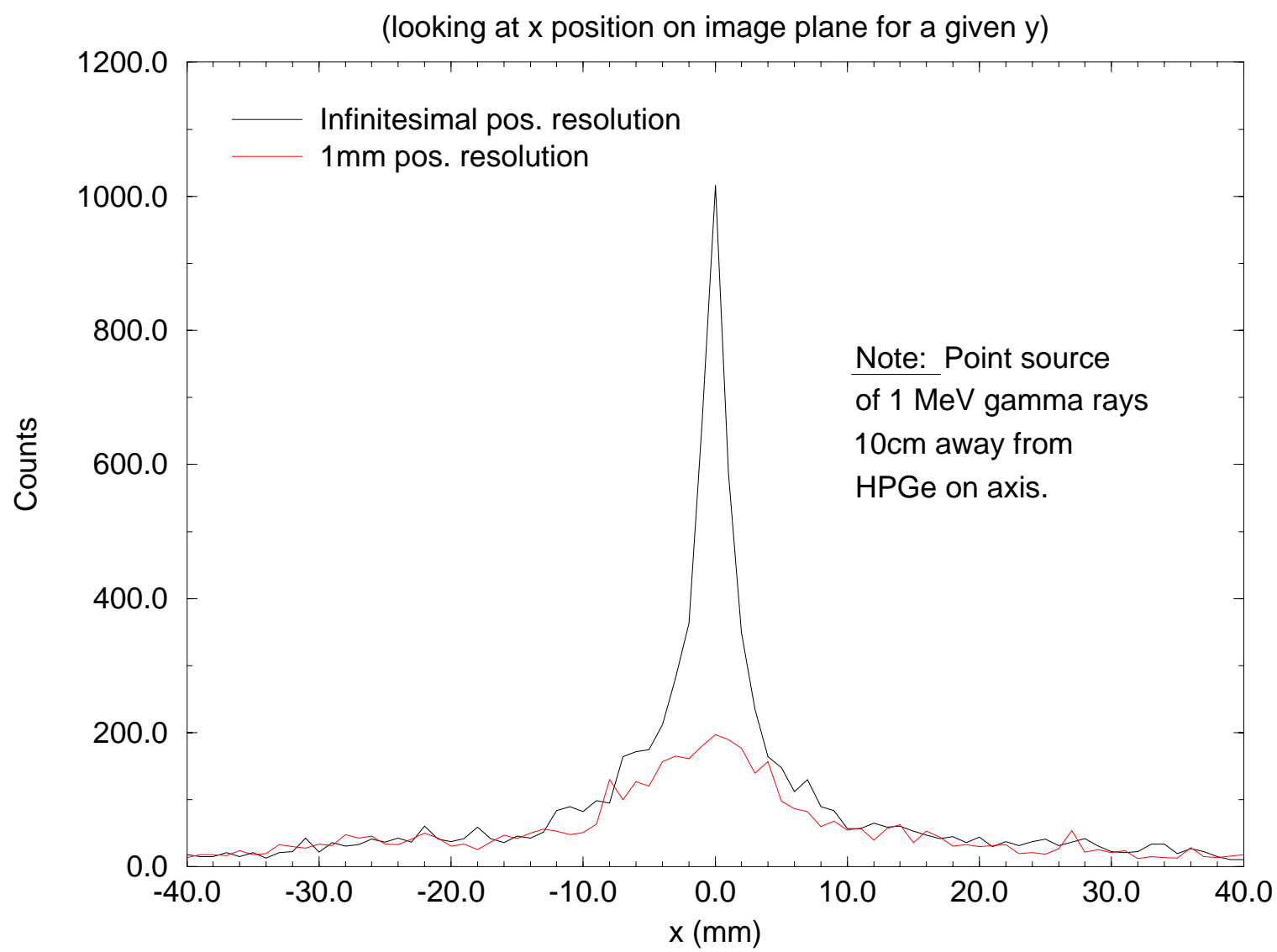

Figure 12: Point response function predicted for a $5 \mathrm{~cm} \times 5 \mathrm{~cm}$ HPGe detector operated as a Compton camera. The black curve assumes infinitesimal position resolution; the red curve assumes 1-mm position resolution. 


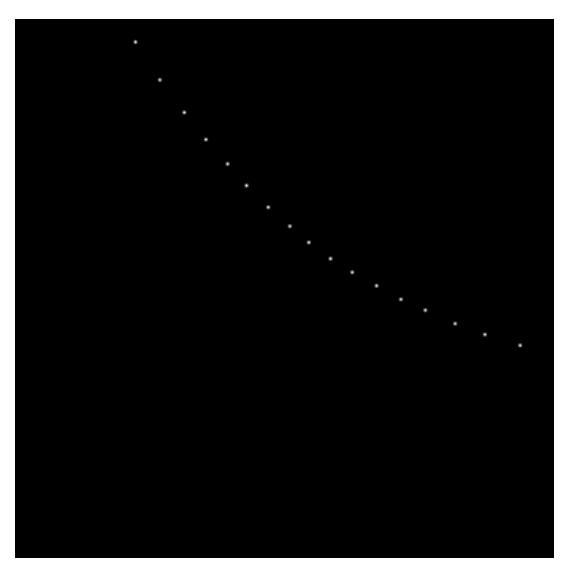

$1 \gamma$-ray

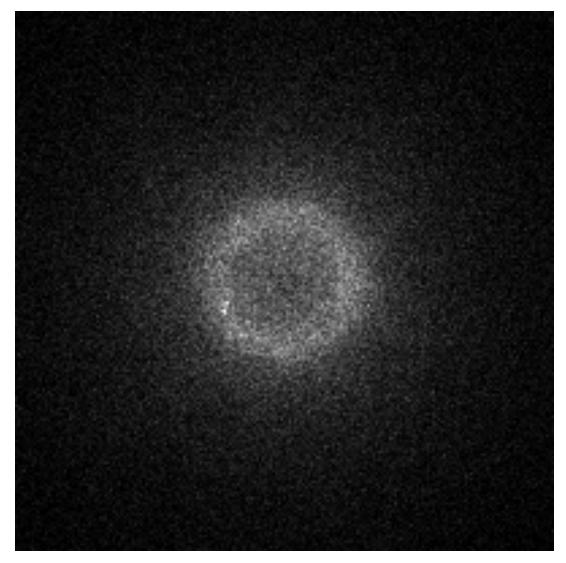

$10,000 \gamma$-rays

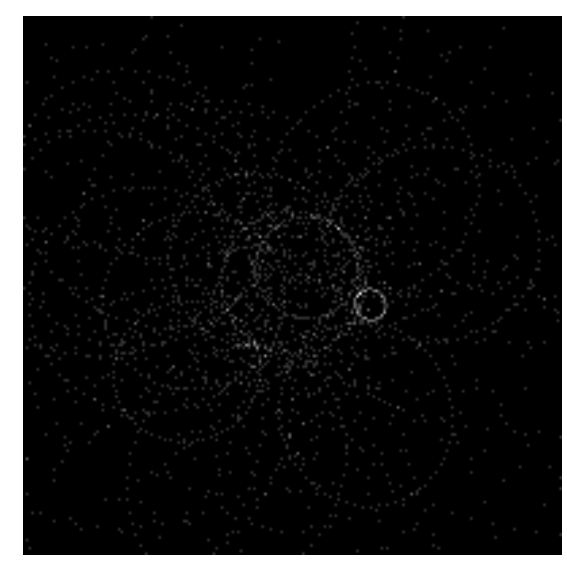

$100 \gamma$-rays

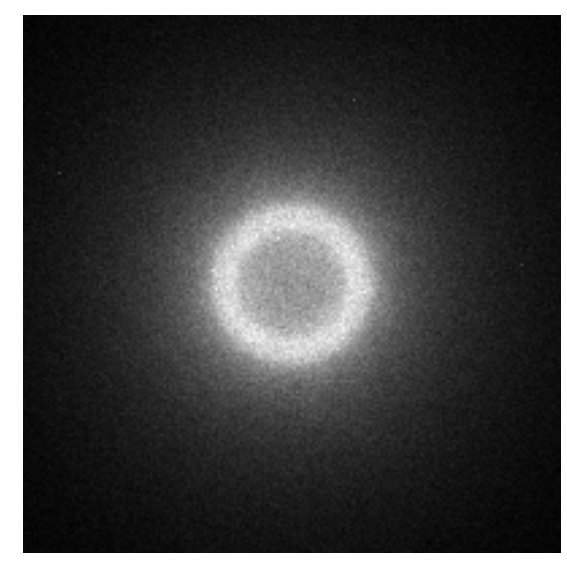

$130,000 \gamma$-rays (+ smoothing)

Figure 13: Evolution of simulated ring image for $5 \mathrm{~cm} \times 5 \mathrm{~cm}$ HPGe detector. The ring, which is assumed to emit $1 \mathrm{MeV} \gamma$-rays, is $6 \mathrm{~cm}$ in diameter and is located $10 \mathrm{~cm}$ away from the detector on axis. Each image consists of the stated number of $\gamma$-ray "probability cones" projected onto the image plane. An infinitesimal HPGe position resolution is assumed. 


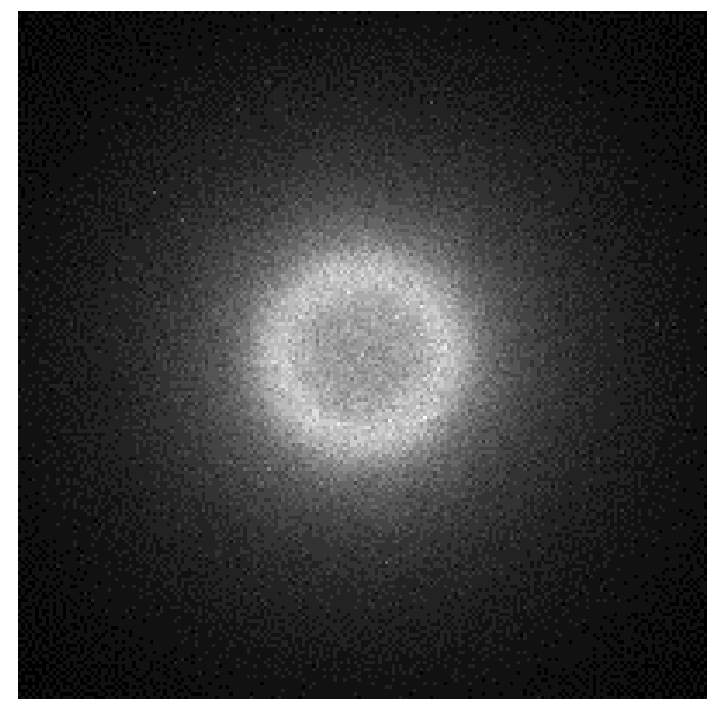

Figure 14: Simulated ring image, corresponding to the lower right figure in figure 13, but obtained with $1 \mathrm{~mm}$ position resolution. 

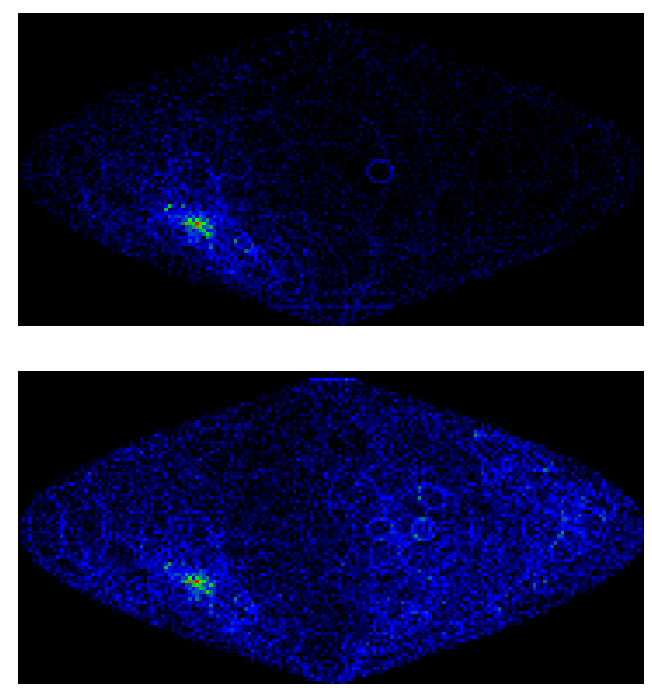

Figure 15: Simulated wide field image plots in a spherical coordinate system centered on the HPGe detector (1m above ground). The abscissa in this plot is $\phi \sin \theta$ and the ordinate is $\theta$. The z-axis of the coordinate system is taken to be parallel to the ground while the positive y direction points "up" (which is to the "left" in these plots). The top picture shows $1 \mathrm{~kg}$ of U235 (located at $\mathrm{r}=3 \mathrm{~m}, \theta=60^{\circ}$, $\phi=90^{\circ}$ ) with no background included. The bottom picture includes $186-\mathrm{keV}$ background radiation coming from the ground. 


\section{Single photon imaging with HPGe}

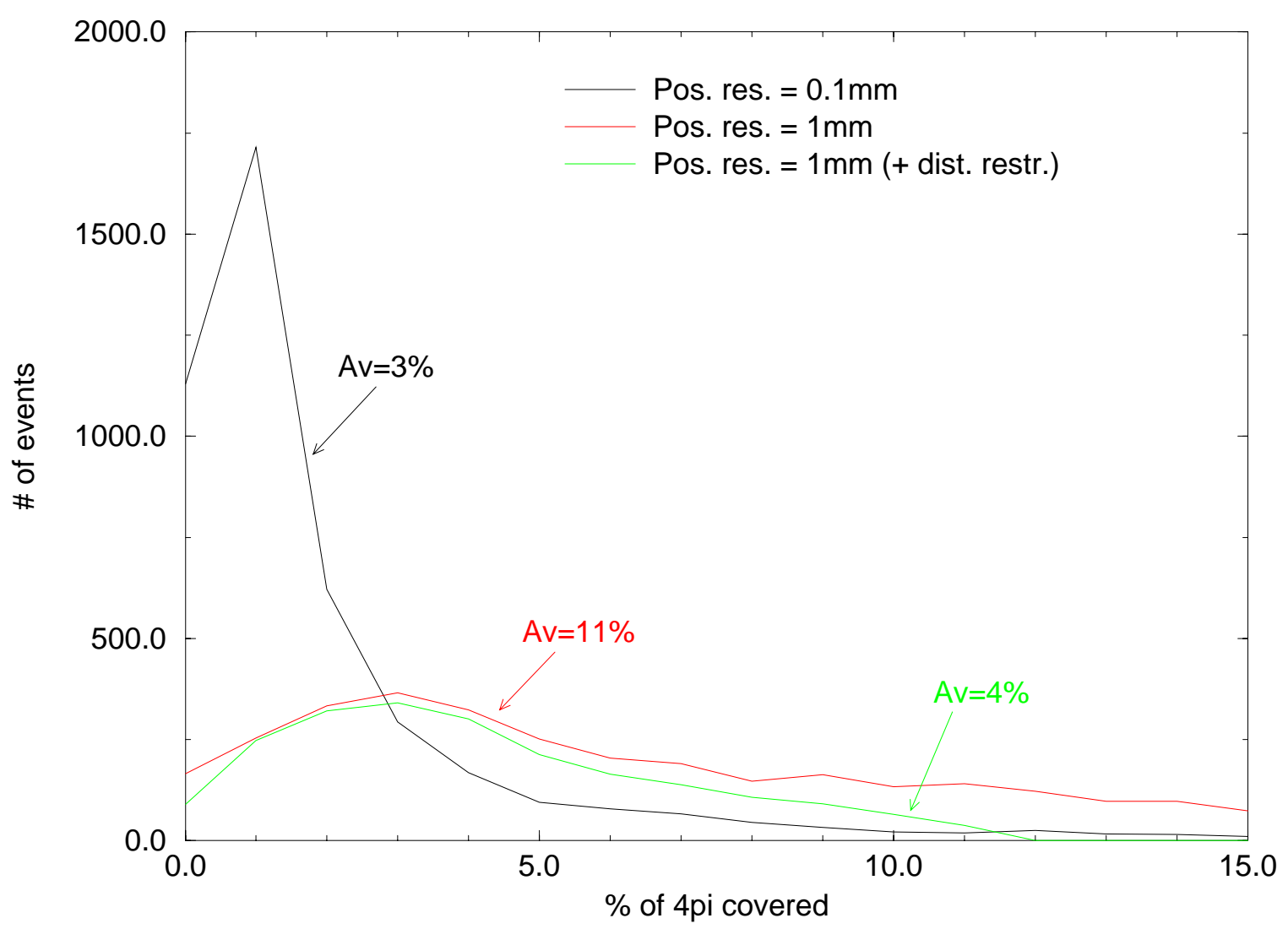

Figure 16: Single photon imaging at $186 \mathrm{keV}$ as a function of position resolution. The energy resolution is taken to be $1 \mathrm{keV}$, but this is not a sensitive parameter. For the green curve, a distance restriction is also imposed, whereby we use only those events in which the first two interaction points are at least $5 \mathrm{~mm}$ apart. This improves the directional determination, but decreases the efficiency by a factor of two. 